EPJ manuscript No.

(will be inserted by the editor)

\title{
Effects of Saving and Spending Patterns on Holding Time Distribution
}

Ning Ding, Ning Xi, and Yougui Wang a

Department of Systems Science, School of Management, Beijing Normal University, Beijing, 100875, P.R. China

Received: date / Revised version: date

\begin{abstract}
The effects of saving and spending patterns on holding time distribution of money are investigated based on the ideal gas-like models. We show the steady-state distribution obeys an exponential law when the saving factor is set uniformly, and a power law when the saving factor is set diversely. The power distribution can also be obtained by proposing a new model where the preferential spending behavior is considered. The association of the distribution with the probability of money to be exchanged has also been discussed.
\end{abstract}

PACS. 89.65.Gh Economics; econophysics, financial markets, business and management - 87.23.Ge Dynamics of social systems - 05.10.-a Computational methods in statistical physics and nonlinear dynamics -02.50 .-r Probability theory, stochastic processes, and statistics

\section{Introduction}

During the last several years, methods and techniques of statistical physics have been successfully applied to economical and financial problems [1,2,3]. Recently, Some econophysists have been paying attention to the statistical mechanics of money, theoretically or empirically [4,5, 6,7, 8 9 10 11, 12. They believe that a thorough understanding of the statistical mechanics of the money, especially Send offprint requests to: Yougui Wang

a E-mail: ygwang@bnu.edu.cn studying of the distribution functions, is essential. Some pioneering work along this line has been reviewed in a popular article 13 .

As well known, the exploration of the distribution of money can be traced back at least a century to the work of the Italian social economist Vilfredo Pareto, who studied the distribution of income among people in different western countries and found an inverse power law 14 . Recently this topic has been taken up with the emergence of econophysicists among whom some believe that there 
might be some physical and mathematical rules governing the distribution of income or wealth in the world and attempt to discover them. A series of models have been developed for the equilibrium money distribution based on the analogy between market economics and kinetic theory of gases 4, 5, 6, 8, 12]. Identifying exchange between any two agents in a closed economy where the money is conserved with the two-body elastic collision in an ideal gas, these models show no matter how uniformly and forcefully one distributes money among agents initially, the successive tradings eventually lead to a steady distribution of money. And the shape of money distribution is determined by the trading rule for choosing an amount of money to transfer. Allowing agents to hold back some of their money when they are chosen to trade, B.K. Chakraborti et al. introduced the saving behavior into the model by adding a saving factor $s$ in the trading rule $[6$. The simulation results clearly indicate a robust Gibbs-like distribution where the density of agents with money $m$ decreases exponentially with $m$ for $s=0$, which is identical to the result of A. Drăgulescu and V. M. Yakovenko's random two-agent exchanges model [5]. The distribution of money changes to follow asymmetric Gibbs-like law when the fixed and uniform saving factor is set to be nonzero, while a 'critical' Pareto distribution of money is found when saving factor is set diversely among agents $6,7,8]$.

In practice, money is held as a store of value, what is more, it plays an essential role for being a medium of exchange. Money is transferred consecutively from hand to hand in the exchange process, in which there exist time intervals for money to be held. This kind of time interval was ever called by Wicksell the "average period of idleness" or "interval of rest" of money [15]. In our previous work [16], we called it "holding time" of money and found that after the economy has achieved an equilibrium state, there is not only a distribution of money among agents, but also a steady distribution over the holding time. We also found that monetary velocity, an important macroeconomic variable, which is associated with Irving Fisher [17, could be expressed as the expectation of the reciprocal of holding time.

In a basic ideal gas-like model, the distribution of money over the holding time follows an exponential law, where saving behavior is not taken into account. The purpose of this paper is to study how the introduction of saving behavior affects such kind of distribution. In next section, we make a brief review of the basic ideal gas-like model by which our work can be erected and of the measurement of the distribution of holding time. In sections 3 and 4. we show that the uniform saving factor gives exponential distribution, while the diverse saving factor induces a change to power distribution. Then we introduce preferential spending behavior into the model in section $[5$ and again obtain power distribution. Comparing these results, we can conclude that the formation of holding time distribution is associated with the character of the probability of money to be exchanged. 


\section{An Ideal Gas-Like Market Model and Holding Time Distribution}

We begin with the basic ideal gas-like model which was introduced firstly by A. Dragulescu and V.M. Yakovenko [5]. A close economy is considered in the model where the amount of money $M$ is conserved and the number of agents $N$ is fixed. The money is possessed by agents individually and agents can exchange money with each other. Since the scale and initial distribution of money have no effect on the final results, most of our simulations were carried out with $N=250$ and $M=25000$ and the amount of money held by each agent was set to be $M / N$ at the beginning. The trade in the economy is modelled to take place round by round. In each round, two agents, $i$ and $j$ for example, are chosen randomly to get engaged in a trade among which agent $i$ is "receiver" and the other one $j$ is "payer". The amount of money that changes hand $\Delta m$ is determined by trading rule which ensures that the amount of any agent's money is non-negative and the total money is conserved. A trading rule commonly used can be expressed as $\Delta m=\varepsilon\left(m_{i}+m_{j}\right) / 2$, where $\varepsilon$ is a random number from zero to unity. As for which units of money are chosen to be transferred, all in the payer's hand is equally probable.

In the ideal gas-like model, money is held by agents and transferred frequently. In this process, if an agent receives money from other agents, he will hold it in hand till paying it out to some other agents. The time interval between the receiving and paying out is named as holding time [16. The holding times of a certain unit of money at different moments or those of different units of money at a given moment are not the same. We introduce the probability distribution function of holding time $P_{h}(\tau)$, which is defined so that the amount of money whose holding time lies between $\tau$ and $\tau+d \tau$ is equal to $M P_{h}(\tau) d \tau$. So, we can get the normalization condition and the expression of the expectation of holding time as follows:

$$
\int_{0}^{\infty} P_{h}(\tau) d \tau=1
$$

and

$$
T=\int_{0}^{\infty} \tau P_{h}(\tau) d \tau .
$$

In the simulations, suppose it is at round $t_{0}$ that we start to reord, and so, holding time is recorded as the difference between the moments when the money takes part in trade after $t_{0}$ for the first two times. The recording mode is illustrated in Figure 1(a). Please note this mode is different from what we have adopted in Reference [16], which is shown in Figure 1(b). The measurement results of the two modes seem quite different, however, they reflect the same process in different ways. We adopt herein the mode (a) solely to facilitate the exposition. The typical distribution of holding time is shown in Figure 2. It can been seen from the inset of Figure 2 that the distribution of holding time follows an exponential law:

$$
P_{h}(\tau)=\frac{1}{T} e^{-\frac{\tau}{T}} .
$$

This result indicates that the transferring process of money is a Poisson process with intensity of $\frac{1}{T}$.

To get the distribution of holding time without systematic factor disturbing, we performed the simulations about 
100 times with different random seeds and data were not collected until the probability distribution of money got stationary. And for convenience we stopped data collecting after majority of money $(>99.9 \%)$ had been recorded. In all the following simulations, holding times are measured in this way, after the distributions of money get stationary of course.

\section{Model with Uniform Saving Factor}

In reality, saving behavior is a natural action pattern for any economic agent. In order to insure future consumption, people always keep a part of their money as saving. The ratio of the saving to total amount of money held by an agent is called "marginal propensity to save" by B. K. Chakrabarti's group. The term of marginal propensity to save has totally different meaning in economics, which is defined as the partial derivative of saving function with respect to income [18. To avoid confusion, we rename it as "saving factor". Referring to the saving factor, two cases have been considered by Chakrabarti's group, one is that all agents have a uniform saving factor, the other one is that saving factors are randomly distributed among agents 6.8. As mentioned above, they found the equilibrium distributions of money among agents had remarkable different characters under such two assumptions. Along this line, in this section and the next one we shall examine the impacts of the saving behavior on the distribution of holding time for the two cases respectively.

All the assumptions of the above ideal gas-like model do work in this model. The amount of money is conserved and the number of agents is fixed. Any agent's money is non-negative or no debt is allowed. The agents are indistinctive at the beginning of simulations: same initial amount of money and same saving factor $s$. In each round, an arbitrary pair of agents are chosen to make exchange with each other. For example, at $t$-th round, agent $i$ and $j$ take part in trading, so that at $t+1$-th round their money $m_{i}(t)$ and $m_{j}(t)$ change to

$$
m_{i}(t+1)=m_{i}(t)+\Delta m ; m_{j}(t+1)=m_{j}(t)-\Delta m ;
$$

where

$$
\Delta m=(1-s)\left[(\varepsilon-1) m_{i}(t)+\varepsilon m_{j}(t)\right] ;
$$

and $\varepsilon$ is a random fraction. After a straight-forward substitution, it is obvious that the trading rule satisfies the conservation and non-negativity condition, and each agent saves fraction $s$ of his money before trade.

The simulation results are shown in Figure 3, for some values of $s$. It can be seen that the probability distributions of holding time for all saving factors decay exponentially. And the lower the saving factor is, the steeper the distribution curve. These results indicate this kind of saving behavior does not change the Poisson nature of the exchanging process, but its intensity.

\section{Model with Diverse Saving Factor}

In realistic economy, how much an agent saves depends on the economic situations he or she faces, and the saving factor of course varies from agent to agent due to their different conditions. To get closer to reality, this model 
inherits all the assumptions and evolution mechanism of the previous model except that of uniform saving factor. Each agent's saving factor is initialized at the beginning of simulations which distributes randomly and uniformly within an interval 0 to 1 , and is fixed in the simulations. Correspondingly, the trading rule Equation (5) changes to

$$
\Delta m=\left(1-s_{i}\right)(\varepsilon-1) m_{i}(t)+\left(1-s_{j}\right) \varepsilon m_{j}(t)
$$

where $s_{i}, s_{j}$ are the saving factors of agent $i$ and $j$ respectively.

To our surprise, once the diverse saving factor is introduced into the model, as shown in Figure 4, the holding time distribution changes to obey a power law instead of an exponential law. This result indicates that the transferring process of money in this model is not a Poisson process any more. The Poisson nature of the process is broken due to the loss of homogeneity of the money transferring. In the previous model, for any saving factor, the probability of each unit of money to participate in exchanges at any round is equal because the saving factor is set to be uniform for all agents. On the contrary, in this model the transferring probability of money is not the same any more due to the diversity of the saving factors. This conclusion was verified by two further measurements on the exchange process.

Firstly, we measured the correlation coefficient of agents' saving factors and the amount of money in their hands. As shown in Figure 5, the correlation coefficient increases sharply at the beginning of simulation, starts to decrease slowly after about 2000th round. The reason of the reduction is that the correlation coefficient can not pick up non-linear associations. We also found the correlation coefficient falls to and keeps at about 0.32 after 500000 th round. Although the value of the correlation coefficient is not high enough, it still implies that the agents with higher saving factors hold more money.

Secondly, we computed the average value of saving factors over total money corresponding to their respective holders after the steady distribution of money among agents had been observed. The value is 0.86 which certifies again that there is more money in the hands of holders with higher saving factors. The average value of saving factors over the money transferred was also computed, its value is about 0.52 . This fact says that the money held by agents with higher saving factor has lower probability to take part in trade. If all money has equal probability, combining with the fact that the agents with higher saving factors hold more money, it can be deduced that the value of this kind of average saving factor should be about 0.86 all the time. Thus, we can conclude that the higher the saving factor of a unit of money's holder, the smaller probability for it to be transferred.

\section{Model with Preferential Spending}

From the previous two models, we can see that different saving patterns lead to different holding time distributions. Especially, when the agents' saving factors are diverse, the probability of money to take part in trade differs. Nevertheless, the probabilities of the money held by the same one agent are equal to each other. This is an implicit assumption in all the simulations which means 
the money is homogenous to any agent. However, it is not the case in real life. As the medium of exchange, money changes hand to hand. In this circulation process, money abrades unavoidably. And when agents make exchange, the payers might spend their money with preference according to the degree of abrasion. As a result, the money is not homogenous for agents. To overcome this unrealistic feature, we proposed a new model which is quite similar to the model with uniform saving factor. The only alteration is that the probability of money chosen to change hand is not equal even if the money is held by the same agent, in other words, that the agents spend money with preference.

In each round, two agents, $i$ and $j$, are chosen randomly to participate in the trade. The amount of money transferred is determined by Equation (5). If agent $i$ is the payer, the probability of money $k$ among $m_{i}$ to be transferred is given by:

$$
p(k)=\frac{l_{k}+1}{\sum_{n=1}^{m_{i}}\left(l_{n}+1\right)}
$$

where $l_{n}$ is the times that money $n$ has participated in the trade since the beginning of simulation. Here, we express the probability with the sum of exchange times and 1 instead of exchange times itself in case that denominator be zero at the beginning of simulations.

The probability distributions of holding time for several different saving factors are recorded after money distributions reach stationary state which are shown in Figure 6. All distributions obey power law, and the only difference is the exponent.
The power distribution arises from the diversity of the probability of money to participate in exchanges. At beginning of our simulation, no money has ever taken part in the trade, thus the probabilities are equal for all money according to Equation (7). After some of money are exchanged randomly, they have higher probabilities and the others have relative lower ones. As the times of exchange increase, this slight diversity of money in the probability will be enlarged till a stable distribution is formed. To see this process from another point of view, the longer for one unit of money to wait, the lower probability for it to be spent. In this way, comparing with the case without preference, some money's holding times get shorter, while some get much longer. Thus the power distribution appears.

We studied the holding time distribution at different times, and found the power distribution is robust. For instance, the holding time distribution for $s=0$ still has the power form even after $t=500000$. Contrarily to this, it is just after $t=1000$ that one can clearly observe the steady distribution.

\section{Conclusions}

In this paper, the effects of saving and spending patterns on the distribution of money over holding time are examined by computer simulations. All the simulations are performed basing on the ideal gas-like models. We consider two kinds of assumptions on saving pattern, one is that all agents have uniform saving factor, the other one is that the saving rates are set randomly distributed among the agents. In the model with uniform saving factor, the distri- 
bution of money over the holding time follows an exponential law, while in the model with diverse saving factor the distribution changes to a power type. We further propose a new trading model where the agents spend money with preference and also get power distribution. The simulation results indicate that the final distribution is determined by the character of the probability that money is chosen to participate in the trade.

\section{Acknowledgments}

We would like to thank Zengru Di and Jinshan Wu for useful comments and discussions. This work was supported by the National Science Foundation of China under Grant No. 70071037.

\section{References}

1. Masanao Aoki, New Approaches to Macroeconomic Modeling, (Cambridge university press, Cambridge, 1996).

2. R. N. Mantegna and H. E. Stanley, An Introduction to Econophysics, (Cambridge university press, Cambridge, 2000).

3. V. M. Yakovenko, arXiv: cond-mat/0302270(2003).

4. S. Ispolatov, P. L. Krapivsky and S. Redner, Eur. Phys. J. B 2 267-276 (1998).

5. A. Drăgulescu and V. M. Yakovenko, Eur. Phys. J. B 17 723-726 (2000).

6. A. Chakraborti and B. K. Chakrabarti, Eur. Phys. J. B 17 $167-170(2000)$.

7. B. K. Chakrabarti and A. Chatterjee, in Application of Econophysics: Proc. 2nd Nikkei Econophysics Sympo- sium, edited by H. Takayasu (Springer, Tpkyo, 2003), Arxiv:cond-mat/0302147

8. A. Chatterjee, B. K. Chakrabarti and S. S. Manna, arXiv: cond-mat/0301289 (2003).

9. A. Chakraborti, Int. J. Mod. Phys. C. 13 1315-1321 (2002).

10. A. Drăgulescu and V. M. Yakovenko, in Modeling of Complex Systems: Seventh Granada Lectures, AIP Conference Proceedings 661 (American Institute of Physics, New York, 2003), p. $180-183$.

11. A. Drăgulescu, and V. M. Yakovenko, Physica A 299, 213 (2001).

12. A. Drăgulescu and V. M. Yakovenko, Eur. Phys. J. B 20 585-589 (2001).

13. B. Hayes, Am. Scientist 90 400-405 (2002).

14. V. Pareto, Cours d'Economie Politique, (Librairie Droz, Geneva, 1987).

15. K. Wicksell, Interest and Prices, (1898), Translated by R. F. Kahn, with an introduction by Bertil Ohlin, (Macmillan, London, 1936).

16. Y. Wang, N. Ding and Li Zhang, Physica A 324 665-677 (2003)

17. I. Fisher, The Purchasing Power of Money, (Macmillan, New York, 1911).

18. O. Blanchard, Macroeconomics, 2nd edn. (Prentice Hall Inc., New Jersey, 2000). 


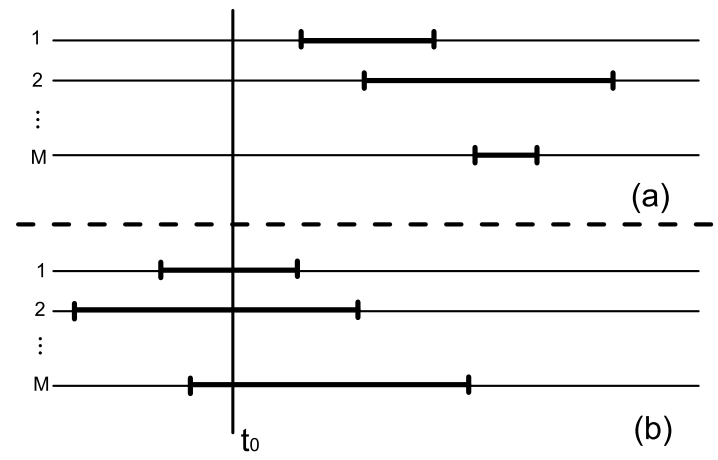

Fig. 1. Schematic presentation of the sampling method of holding time adopted: (a) in this paper; (b) in Reference [16]. $t_{0}$ denotes the sampling time point, the light horizontal solid lines represent the evolution history of money, the vertical short bars symbolize moments for corresponding money to be transferred and then the dark segments correspond to the holding times to be recorded.

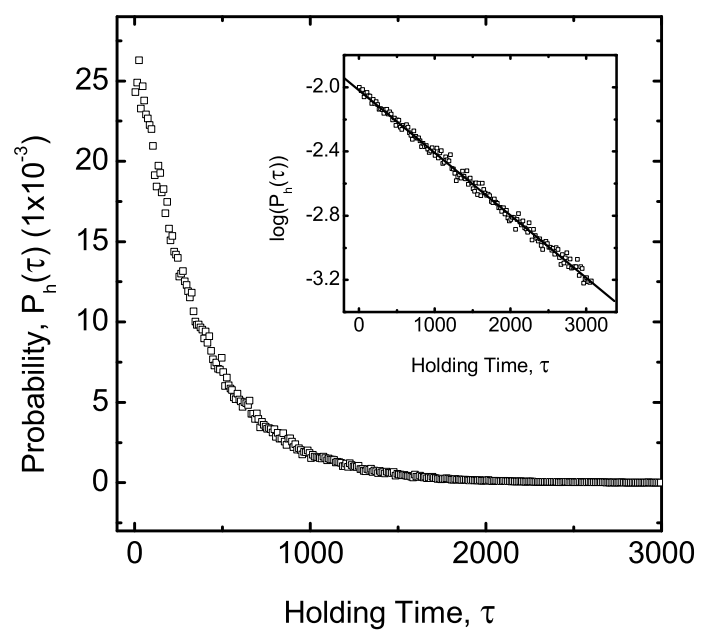

Fig. 2. The stationary holding time distribution obtained from the basic ideal gas-like model simulations versus holding time. The fitting in the inset indicates the distribution follows the exponential law: $P_{h}(\tau)=\frac{1}{T} \exp (-\tau / T)$.

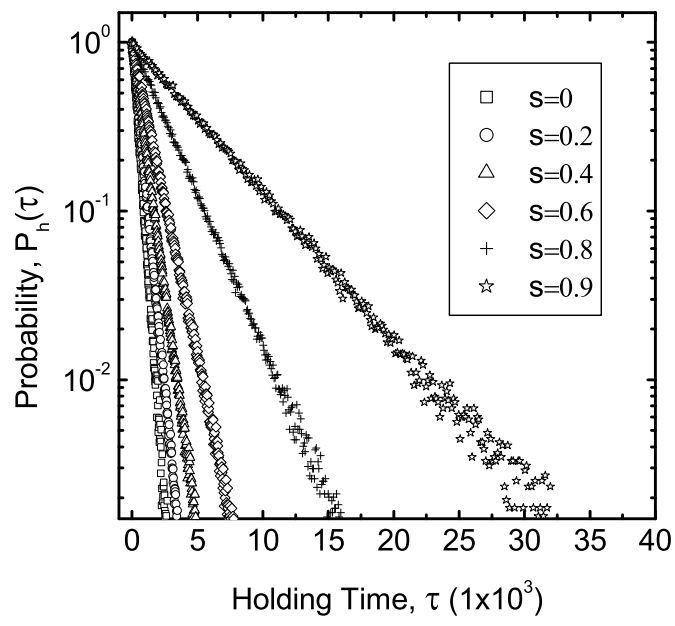

Fig. 3. The stationary distributions of holding time for several saving factors from 0 to 0.9 derived from the simulations of the model with uniform saving factor in the semi-logarithmic scale. Note that in the figure the probabilities have been scaled by the maximum probability respectively.

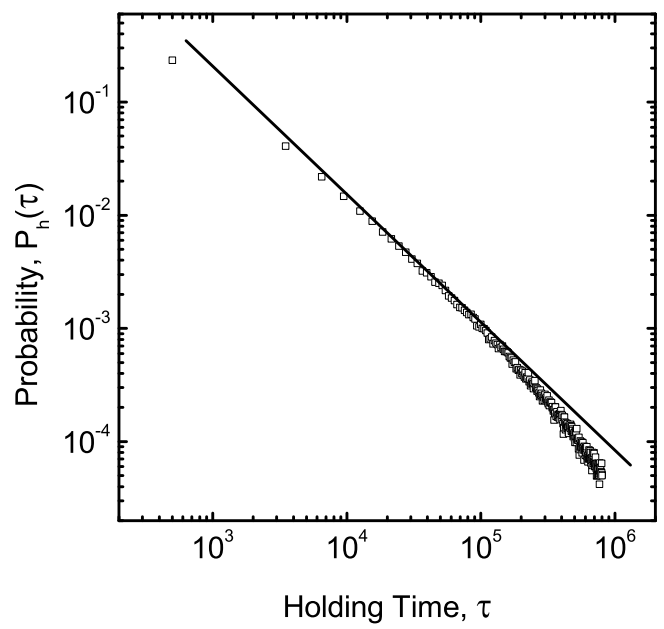

Fig. 4. The stationary distribution of holding time derived from the simulations of the model with diverse saving factor in double logarithmic scale. The solid line is numerically fitted line in the form of $P_{h}(\tau) \propto \tau^{-1.14}$. 


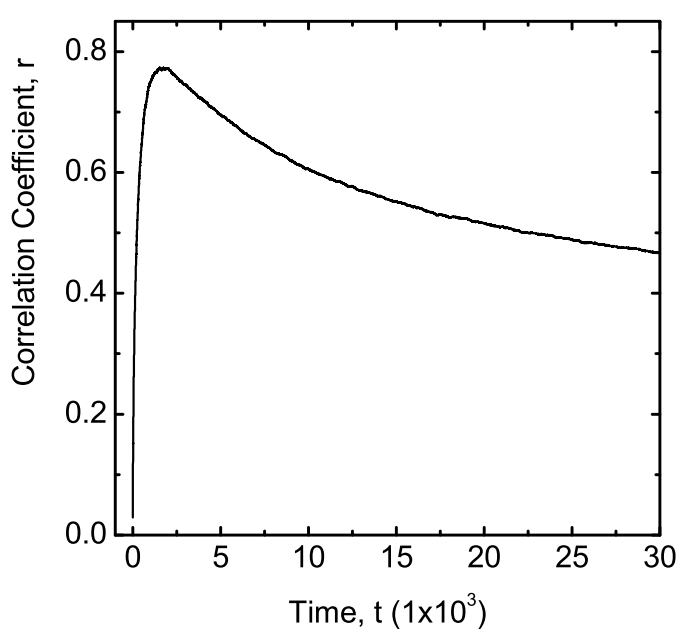

Fig. 5. Correlative coefficient between the amount of money held by agents and their saving factors versus time. At time $t=1541$, the coefficient reaches its maximum 0.773 .

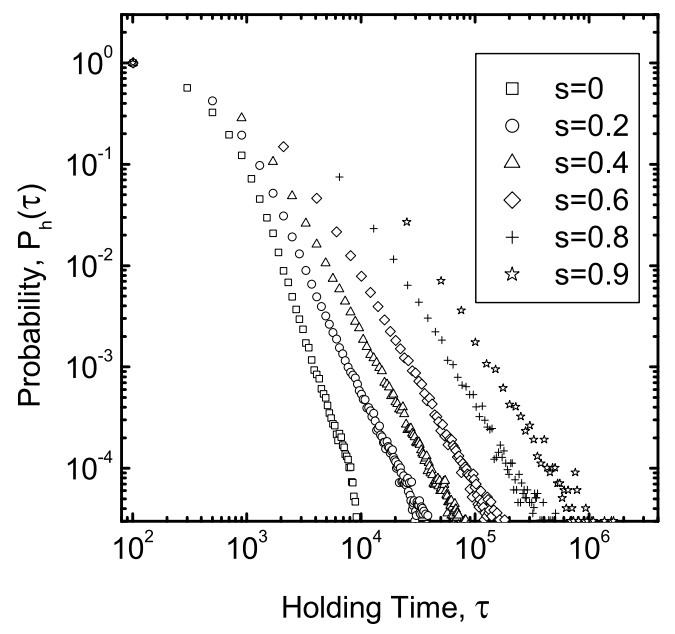

Fig. 6. The stationary distributions of holding time for several uniform saving factors from 0 to 0.9 derived from the simulations of the model with preferential spending in the double logarithmic scale. Note that in the figure the probabilities have been scaled by the maximum probability respectively. 
EPJ manuscript No.

(will be inserted by the editor)

\title{
Effects of Saving and Spending Patterns on Holding Time Distribution
}

Ning Ding, Ning Xi, and Yougui Wang a

Department of Systems Science, School of Management, Beijing Normal University, Beijing, 100875, P.R. China

Received: date / Revised version: date

\begin{abstract}
The effects of saving and spending patterns on holding time distribution of money are investigated based on the ideal gas-like models. We show the steady-state distribution obeys an exponential law when the saving factor is set uniformly, and a power law when the saving factor is set diversely. The power distribution can also be obtained by proposing a new model where the preferential spending behavior is considered. The association of the distribution with the probability of money to be exchanged has also been discussed.
\end{abstract}

PACS. 89.65.Gh Economics; econophysics, financial markets, business and management - 87.23.Ge Dynamics of social systems - 05.10.-a Computational methods in statistical physics and nonlinear dynamics -02.50 .-r Probability theory, stochastic processes, and statistics

\section{Introduction}

During the last several years, methods and techniques of statistical physics have been successfully applied to economical and financial problems [1,2,3]. Recently, Some econophysists have been paying attention to the statistical mechanics of money, theoretically or empirically [4,5, 6,7, 8 9 10 11, 12. They believe that a thorough understanding of the statistical mechanics of the money, especially Send offprint requests to: Yougui Wang

a E-mail: ygwang@bnu.edu.cn studying of the distribution functions, is essential. Some pioneering work along this line has been reviewed in a popular article 13 .

As well known, the exploration of the distribution of money can be traced back at least a century to the work of the Italian social economist Vilfredo Pareto, who studied the distribution of income among people in different western countries and found an inverse power law 14 . Recently this topic has been taken up with the emergence of econophysicists among whom some believe that there 
might be some physical and mathematical rules governing the distribution of income or wealth in the world and attempt to discover them. A series of models have been developed for the equilibrium money distribution based on the analogy between market economics and kinetic theory of gases 4, 5, 6, 8, 12]. Identifying exchange between any two agents in a closed economy where the money is conserved with the two-body elastic collision in an ideal gas, these models show no matter how uniformly and forcefully one distributes money among agents initially, the successive tradings eventually lead to a steady distribution of money. And the shape of money distribution is determined by the trading rule for choosing an amount of money to transfer. Allowing agents to hold back some of their money when they are chosen to trade, B.K. Chakraborti et al. introduced the saving behavior into the model by adding a saving factor $s$ in the trading rule $[6$. The simulation results clearly indicate a robust Gibbs-like distribution where the density of agents with money $m$ decreases exponentially with $m$ for $s=0$, which is identical to the result of A. Drăgulescu and V. M. Yakovenko's random two-agent exchanges model [5]. The distribution of money changes to follow asymmetric Gibbs-like law when the fixed and uniform saving factor is set to be nonzero, while a 'critical' Pareto distribution of money is found when saving factor is set diversely among agents $6,7,8]$.

In practice, money is held as a store of value, what is more, it plays an essential role for being a medium of exchange. Money is transferred consecutively from hand to hand in the exchange process, in which there exist time intervals for money to be held. This kind of time interval was ever called by Wicksell the "average period of idleness" or "interval of rest" of money [15]. In our previous work [16], we called it "holding time" of money and found that after the economy has achieved an equilibrium state, there is not only a distribution of money among agents, but also a steady distribution over the holding time. We also found that monetary velocity, an important macroeconomic variable, which is associated with Irving Fisher [17, could be expressed as the expectation of the reciprocal of holding time.

In a basic ideal gas-like model, the distribution of money over the holding time follows an exponential law, where saving behavior is not taken into account. The purpose of this paper is to study how the introduction of saving behavior affects such kind of distribution. In next section, we make a brief review of the basic ideal gas-like model by which our work can be erected and of the measurement of the distribution of holding time. In sections 3 and 4. we show that the uniform saving factor gives exponential distribution, while the diverse saving factor induces a change to power distribution. Then we introduce preferential spending behavior into the model in section $[5$ and again obtain power distribution. Comparing these results, we can conclude that the formation of holding time distribution is associated with the character of the probability of money to be exchanged. 


\section{An Ideal Gas-Like Market Model and Holding Time Distribution}

We begin with the basic ideal gas-like model which was introduced firstly by A. Dragulescu and V.M. Yakovenko [5]. A close economy is considered in the model where the amount of money $M$ is conserved and the number of agents $N$ is fixed. The money is possessed by agents individually and agents can exchange money with each other. Since the scale and initial distribution of money have no effect on the final results, most of our simulations were carried out with $N=250$ and $M=25000$ and the amount of money held by each agent was set to be $M / N$ at the beginning. The trade in the economy is modelled to take place round by round. In each round, two agents, $i$ and $j$ for example, are chosen randomly to get engaged in a trade among which agent $i$ is "receiver" and the other one $j$ is "payer". The amount of money that changes hand $\Delta m$ is determined by trading rule which ensures that the amount of any agent's money is non-negative and the total money is conserved. A trading rule commonly used can be expressed as $\Delta m=\varepsilon\left(m_{i}+m_{j}\right) / 2$, where $\varepsilon$ is a random number from zero to unity. As for which units of money are chosen to be transferred, all in the payer's hand is equally probable.

In the ideal gas-like model, money is held by agents and transferred frequently. In this process, if an agent receives money from other agents, he will hold it in hand till paying it out to some other agents. The time interval between the receiving and paying out is named as holding time [16. The holding times of a certain unit of money at different moments or those of different units of money at a given moment are not the same. We introduce the probability distribution function of holding time $P_{h}(\tau)$, which is defined so that the amount of money whose holding time lies between $\tau$ and $\tau+d \tau$ is equal to $M P_{h}(\tau) d \tau$. So, we can get the normalization condition and the expression of the expectation of holding time as follows:

$$
\int_{0}^{\infty} P_{h}(\tau) d \tau=1
$$

and

$$
T=\int_{0}^{\infty} \tau P_{h}(\tau) d \tau .
$$

In the simulations, suppose it is at round $t_{0}$ that we start to reord, and so, holding time is recorded as the difference between the moments when the money takes part in trade after $t_{0}$ for the first two times. The recording mode is illustrated in Figure 1(a). Please note this mode is different from what we have adopted in Reference [16], which is shown in Figure 1(b). The measurement results of the two modes seem quite different, however, they reflect the same process in different ways. We adopt herein the mode (a) solely to facilitate the exposition. The typical distribution of holding time is shown in Figure 2. It can been seen from the inset of Figure 2 that the distribution of holding time follows an exponential law:

$$
P_{h}(\tau)=\frac{1}{T} e^{-\frac{\tau}{T}} .
$$

This result indicates that the transferring process of money is a Poisson process with intensity of $\frac{1}{T}$.

To get the distribution of holding time without systematic factor disturbing, we performed the simulations about 
100 times with different random seeds and data were not collected until the probability distribution of money got stationary. And for convenience we stopped data collecting after majority of money $(>99.9 \%)$ had been recorded. In all the following simulations, holding times are measured in this way, after the distributions of money get stationary of course.

\section{Model with Uniform Saving Factor}

In reality, saving behavior is a natural action pattern for any economic agent. In order to insure future consumption, people always keep a part of their money as saving. The ratio of the saving to total amount of money held by an agent is called "marginal propensity to save" by B. K. Chakrabarti's group. The term of marginal propensity to save has totally different meaning in economics, which is defined as the partial derivative of saving function with respect to income [18. To avoid confusion, we rename it as "saving factor". Referring to the saving factor, two cases have been considered by Chakrabarti's group, one is that all agents have a uniform saving factor, the other one is that saving factors are randomly distributed among agents 6.8. As mentioned above, they found the equilibrium distributions of money among agents had remarkable different characters under such two assumptions. Along this line, in this section and the next one we shall examine the impacts of the saving behavior on the distribution of holding time for the two cases respectively.

All the assumptions of the above ideal gas-like model do work in this model. The amount of money is conserved and the number of agents is fixed. Any agent's money is non-negative or no debt is allowed. The agents are indistinctive at the beginning of simulations: same initial amount of money and same saving factor $s$. In each round, an arbitrary pair of agents are chosen to make exchange with each other. For example, at $t$-th round, agent $i$ and $j$ take part in trading, so that at $t+1$-th round their money $m_{i}(t)$ and $m_{j}(t)$ change to

$$
m_{i}(t+1)=m_{i}(t)+\Delta m ; m_{j}(t+1)=m_{j}(t)-\Delta m ;
$$

where

$$
\Delta m=(1-s)\left[(\varepsilon-1) m_{i}(t)+\varepsilon m_{j}(t)\right] ;
$$

and $\varepsilon$ is a random fraction. After a straight-forward substitution, it is obvious that the trading rule satisfies the conservation and non-negativity condition, and each agent saves fraction $s$ of his money before trade.

The simulation results are shown in Figure 3, for some values of $s$. It can be seen that the probability distributions of holding time for all saving factors decay exponentially. And the lower the saving factor is, the steeper the distribution curve. These results indicate this kind of saving behavior does not change the Poisson nature of the exchanging process, but its intensity.

\section{Model with Diverse Saving Factor}

In realistic economy, how much an agent saves depends on the economic situations he or she faces, and the saving factor of course varies from agent to agent due to their different conditions. To get closer to reality, this model 
inherits all the assumptions and evolution mechanism of the previous model except that of uniform saving factor. Each agent's saving factor is initialized at the beginning of simulations which distributes randomly and uniformly within an interval 0 to 1 , and is fixed in the simulations. Correspondingly, the trading rule Equation (5) changes to

$$
\Delta m=\left(1-s_{i}\right)(\varepsilon-1) m_{i}(t)+\left(1-s_{j}\right) \varepsilon m_{j}(t)
$$

where $s_{i}, s_{j}$ are the saving factors of agent $i$ and $j$ respectively.

To our surprise, once the diverse saving factor is introduced into the model, as shown in Figure 4, the holding time distribution changes to obey a power law instead of an exponential law. This result indicates that the transferring process of money in this model is not a Poisson process any more. The Poisson nature of the process is broken due to the loss of homogeneity of the money transferring. In the previous model, for any saving factor, the probability of each unit of money to participate in exchanges at any round is equal because the saving factor is set to be uniform for all agents. On the contrary, in this model the transferring probability of money is not the same any more due to the diversity of the saving factors. This conclusion was verified by two further measurements on the exchange process.

Firstly, we measured the correlation coefficient of agents' saving factors and the amount of money in their hands. As shown in Figure 5, the correlation coefficient increases sharply at the beginning of simulation, starts to decrease slowly after about 2000th round. The reason of the reduction is that the correlation coefficient can not pick up non-linear associations. We also found the correlation coefficient falls to and keeps at about 0.32 after 500000 th round. Although the value of the correlation coefficient is not high enough, it still implies that the agents with higher saving factors hold more money.

Secondly, we computed the average value of saving factors over total money corresponding to their respective holders after the steady distribution of money among agents had been observed. The value is 0.86 which certifies again that there is more money in the hands of holders with higher saving factors. The average value of saving factors over the money transferred was also computed, its value is about 0.52 . This fact says that the money held by agents with higher saving factor has lower probability to take part in trade. If all money has equal probability, combining with the fact that the agents with higher saving factors hold more money, it can be deduced that the value of this kind of average saving factor should be about 0.86 all the time. Thus, we can conclude that the higher the saving factor of a unit of money's holder, the smaller probability for it to be transferred.

\section{Model with Preferential Spending}

From the previous two models, we can see that different saving patterns lead to different holding time distributions. Especially, when the agents' saving factors are diverse, the probability of money to take part in trade differs. Nevertheless, the probabilities of the money held by the same one agent are equal to each other. This is an implicit assumption in all the simulations which means 
the money is homogenous to any agent. However, it is not the case in real life. As the medium of exchange, money changes hand to hand. In this circulation process, money abrades unavoidably. And when agents make exchange, the payers might spend their money with preference according to the degree of abrasion. As a result, the money is not homogenous for agents. To overcome this unrealistic feature, we proposed a new model which is quite similar to the model with uniform saving factor. The only alteration is that the probability of money chosen to change hand is not equal even if the money is held by the same agent, in other words, that the agents spend money with preference.

In each round, two agents, $i$ and $j$, are chosen randomly to participate in the trade. The amount of money transferred is determined by Equation (5). If agent $i$ is the payer, the probability of money $k$ among $m_{i}$ to be transferred is given by:

$$
p(k)=\frac{l_{k}+1}{\sum_{n=1}^{m_{i}}\left(l_{n}+1\right)}
$$

where $l_{n}$ is the times that money $n$ has participated in the trade since the beginning of simulation. Here, we express the probability with the sum of exchange times and 1 instead of exchange times itself in case that denominator be zero at the beginning of simulations.

The probability distributions of holding time for several different saving factors are recorded after money distributions reach stationary state which are shown in Figure 6. All distributions obey power law, and the only difference is the exponent.
The power distribution arises from the diversity of the probability of money to participate in exchanges. At beginning of our simulation, no money has ever taken part in the trade, thus the probabilities are equal for all money according to Equation (7). After some of money are exchanged randomly, they have higher probabilities and the others have relative lower ones. As the times of exchange increase, this slight diversity of money in the probability will be enlarged till a stable distribution is formed. To see this process from another point of view, the longer for one unit of money to wait, the lower probability for it to be spent. In this way, comparing with the case without preference, some money's holding times get shorter, while some get much longer. Thus the power distribution appears.

We studied the holding time distribution at different times, and found the power distribution is robust. For instance, the holding time distribution for $s=0$ still has the power form even after $t=500000$. Contrarily to this, it is just after $t=1000$ that one can clearly observe the steady distribution.

\section{Conclusions}

In this paper, the effects of saving and spending patterns on the distribution of money over holding time are examined by computer simulations. All the simulations are performed basing on the ideal gas-like models. We consider two kinds of assumptions on saving pattern, one is that all agents have uniform saving factor, the other one is that the saving rates are set randomly distributed among the agents. In the model with uniform saving factor, the distri- 
bution of money over the holding time follows an exponential law, while in the model with diverse saving factor the distribution changes to a power type. We further propose a new trading model where the agents spend money with preference and also get power distribution. The simulation results indicate that the final distribution is determined by the character of the probability that money is chosen to participate in the trade.

\section{Acknowledgments}

We would like to thank Zengru Di and Jinshan Wu for useful comments and discussions. This work was supported by the National Science Foundation of China under Grant No. 70071037.

\section{References}

1. Masanao Aoki, New Approaches to Macroeconomic Modeling, (Cambridge university press, Cambridge, 1996).

2. R. N. Mantegna and H. E. Stanley, An Introduction to Econophysics, (Cambridge university press, Cambridge, 2000).

3. V. M. Yakovenko, arXiv: cond-mat/0302270

4. S. Ispolatov, P. L. Krapivsky and S. Redner, Eur. Phys. J. B 2 267-276 (1998).

5. A. Drăgulescu and V. M. Yakovenko, Eur. Phys. J. B 17 723-726 (2000).

6. A. Chakraborti and B. K. Chakrabarti, Eur. Phys. J. B 17 $167-170(2000)$.

7. B. K. Chakrabarti and A. Chatterjee, in Application of Econophysics: Proc. 2nd Nikkei Econophysics Sympo- sium, edited by H. Takayasu (Springer, Tpkyo, 2003), cond-mat/0302147

8. A. Chatterjee, B. K. Chakrabarti and S. S. Manna, arXiv: cond-mat/0301289

9. A. Chakraborti, Int. J. Mod. Phys. C. 13 1315-1321 (2002).

10. A. Drăgulescu and V. M. Yakovenko, in Modeling of Complex Systems: Seventh Granada Lectures, AIP Conference Proceedings 661 (American Institute of Physics, New York, 2003), p. $180-183$.

11. A. Drăgulescu, and V. M. Yakovenko, Physica A 299, 213 (2001).

12. A. Drăgulescu and V. M. Yakovenko, Eur. Phys. J. B 20 585-589 (2001).

13. B. Hayes, Am. Scientist 90 400-405 (2002).

14. V. Pareto, Cours d'Economie Politique, (Librairie Droz, Geneva, 1987).

15. K. Wicksell, Interest and Prices, (1898), Translated by R. F. Kahn, with an introduction by Bertil Ohlin, (Macmillan, London, 1936).

16. Y. Wang, N. Ding and Li Zhang, Physica A 324 665-677 (2003)

17. I. Fisher, The Purchasing Power of Money, (Macmillan, New York, 1911).

18. O. Blanchard, Macroeconomics, 2nd edn. (Prentice Hall Inc., New Jersey, 2000). 


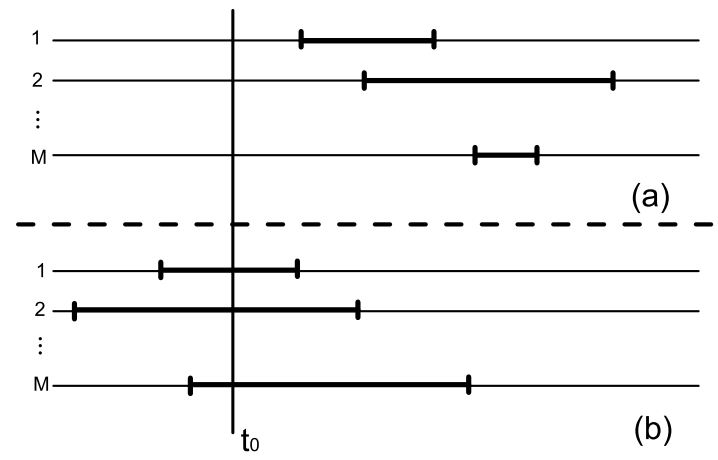

Fig. 1. Schematic presentation of the sampling method of holding time adopted: (a) in this paper; (b) in Reference [16]. $t_{0}$ denotes the sampling time point, the light horizontal solid lines represent the evolution history of money, the vertical short bars symbolize moments for corresponding money to be transferred and then the dark segments correspond to the holding times to be recorded.

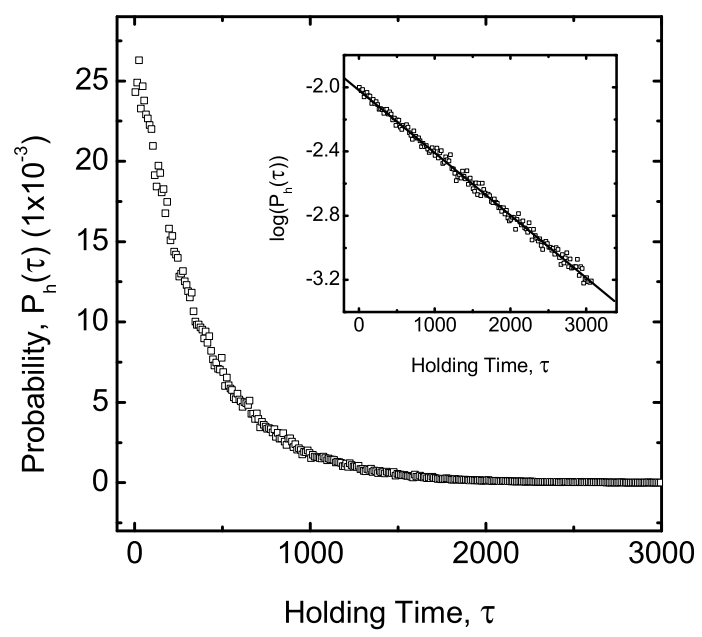

Fig. 2. The stationary holding time distribution obtained from the basic ideal gas-like model simulations versus holding time. The fitting in the inset indicates the distribution follows the exponential law: $P_{h}(\tau)=\frac{1}{T} \exp (-\tau / T)$.

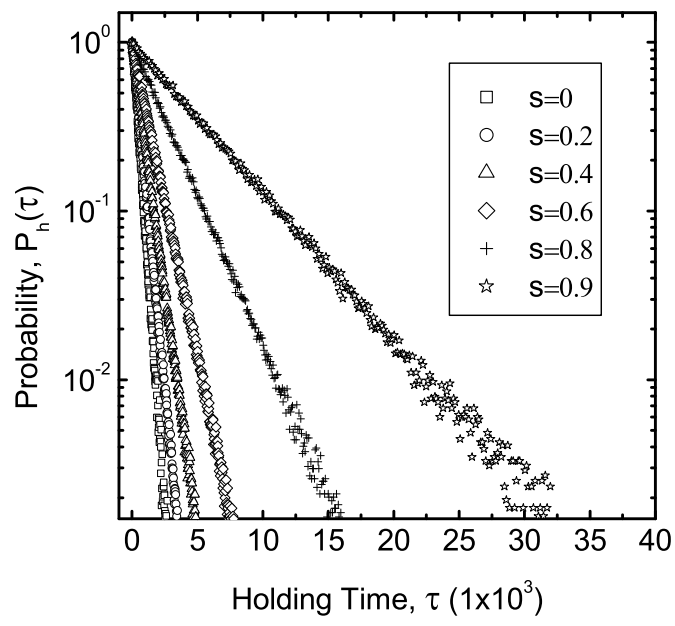

Fig. 3. The stationary distributions of holding time for several saving factors from 0 to 0.9 derived from the simulations of the model with uniform saving factor in the semi-logarithmic scale. Note that in the figure the probabilities have been scaled by the maximum probability respectively.

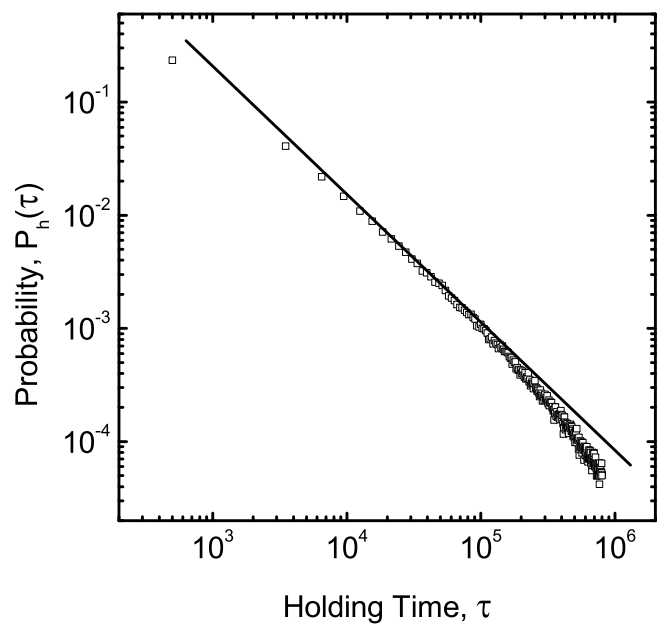

Fig. 4. The stationary distribution of holding time derived from the simulations of the model with diverse saving factor in double logarithmic scale. The solid line is numerically fitted line in the form of $P_{h}(\tau) \propto \tau^{-1.14}$. 


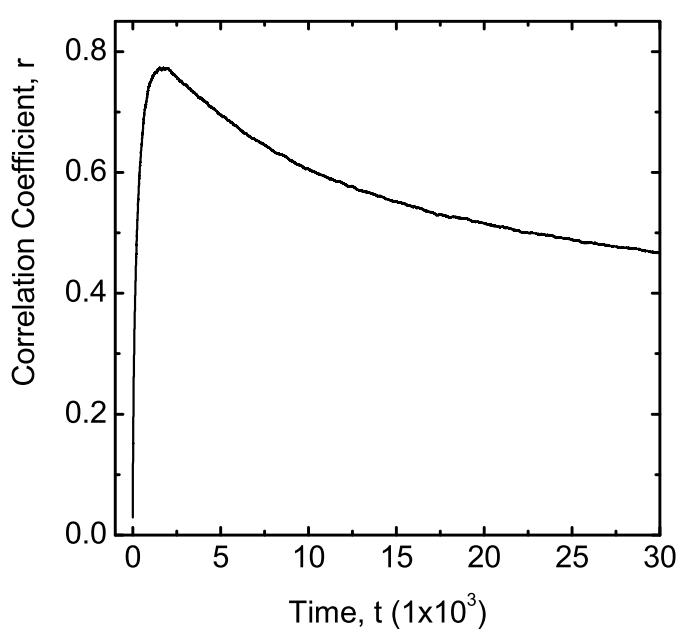

Fig. 5. Correlative coefficient between the amount of money held by agents and their saving factors versus time. At time $t=1541$, the coefficient reaches its maximum 0.773 .

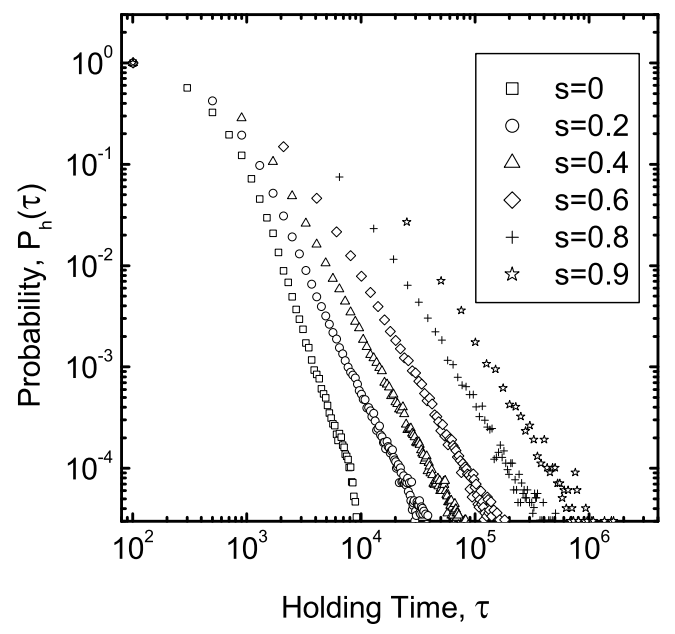

Fig. 6. The stationary distributions of holding time for several uniform saving factors from 0 to 0.9 derived from the simulations of the model with preferential spending in the double logarithmic scale. Note that in the figure the probabilities have been scaled by the maximum probability respectively. 